\title{
Assessing the Topological Consistency of Crowdsourced OpenStreetMap Data
}

\author{
SUKHJIT SINGH SEHRA Assistant Professor, Guru Nanak Dev Engineering College, Ludhiana, India
}

JAITEG SINGH, Assistant Professor, Chitkara Institute of Engineering \& Technology, Punjab, India

HARDEEP SINGH RAI, Professor, Guru Nanak Dev Engineering College, Ludhiana, India

\begin{abstract}
Despite the scale-related advantages of online crowdsourcing, human computation systems are prone to human-based errors. Fortunately, machines can be used in complementary fashion to detect and correct those errors either autonomously or by enlisting the help of humans during key steps. Herein we consider the relevant case of OpenStreetMap, which is a world leader in collecting map data contributed by users. We have little knowledge about its contributors in terms of their skills, knowledge, and patterns of data collection. Furthermore, OpenStreetMap has loose coordination and no top-down quality assurance processes. This makes the crowdsourced map data more vulnerable to errors and gaps, which would need to be corrected in order for the resultant map to be navigable. The present work has been conducted to identify errors in OpenStreetMap data, using a metropolitan area in Punjab as test data for finding inconsistencies. We conclude that our test data contains many such errors and is not mature enough to support practical use. We also describe various open-source algorithms that could be used remedially to address these shortcomings.
\end{abstract}

\section{INTRODUCTION}

Maps have been an integral part of mankind for a long time. From cave paintings through the $21^{\text {st }}$ century, people have created and used maps as an essential tool to help them define, explain, and navigate their ways through the world. Recently, mapping technologies began to be used in the car for navigation. Today, mapping enables everything from lucrative location-based services to game-changing autonomous driving (Newcomb, 2014). 
Modern cartography has been strongly integrated with Geographical Information Systems. Earlier cartography was a specialised job, but the evolution of the Web made it possible that noncommercialised people can now also contribute. The Web started with web 1.0 or "read only" web, but Tim (O'Reilly, 2005) discussed the concept of Web 2.0 or "read-write" web. Client side technologies such as Ajax and Javascript frameworks are now used heavily for Web 2.0 development ( $\mathrm{O}^{`}$ Reilly, 2005). Web 2.0 encourages greater collaboration among Internet users, content providers, and enterprises (Hudson-Smith et al., 2009). This movement provides revolutionary new methods of sharing and computing data (Hudson-Smith et al., 2009; Turner, 2006; Goodchild, 2009; Haklay et al., 2008) provided by crowdsourcing efforts similar to Wikipedia (Howe, 2006). In regard to the geographical data, the crowd-sourced movement is known as VGI (volunteered geographic information), also named Neography in regard to web 2.0 (Goodchild, 2009; Haklay et al., 2008) and collaborative mapping (Fischer, 2008), so it is a special case of this web phenomenon and has been applied in many popular websites such as: Wikimapia, OpenStreetMap (OSM), Googlemap, Flickr (Kounadi, 2009).

On the other side, the advent of inexpensive portable satellite navigation devices and GPS enabled smartphones helped users to contribute to crowdsourced OpenStreetMap. The accuracy of smartphones based on iOS, Android, Windows Mobile, BlackBerry OS and Symbian mobile operating systems has been already tested and verified (Golicher, 2013). As of December 2012, the worldwide smartphone market had Android as its top operating system, counting on $68.3 \%$ of the market share, followed by iOS with $18.8 \%$ and Blackberry with $4.7 \%$. Forecasts show that by 2016 the Windows Phone operating system will overtake third place, with $11.4 \%$ of the market share, while Android and iOS will remain in its previous positions, with $63.8 \%$ and $19.1 \%$ respectively. All of these mobile phones are not only used for calling, but are built standard with many applications. These applications are used for navigation and include location based service applications which enable geotagging. According to one survey, Indians are more sophisticated users of Smartphones than American users as per a survey report published by Google along with IPSOS and MMA (Mobile Marketing Association). The survey declares that, for many Indians, Smart phones are not just a medium to call or message but a smart device to meet their multiple needs such as navigation, social networking, share market etc.

As geography is big business, map data providers such as Google, NavTeq, Mapmyindia, TomTom and TeleAtlas are in a race to acquire world map data because such companies realize that non-geospatial applications are integrated with spatial data. But there is a huge cost involved in map production (e.g. Navteq is spending approximately $\$ 400 \mathrm{~m}$ in creation and distribution of maps and has nearly a 3,400 person work force). In addition, big companies have invested large sums of money to purchase smaller companies to acquire their map data (e.g., in 2007, Nokia acquired NavTeq, in 2006, Microsoft acquired the Imagery and Remote Sensing Company Vexcel (Schmitz et al., 2008) and in 2014 acquired waze for \$1.5billion because waze was producing a very large volume of user generated map content.

In 2004, to provide freely editable maps of the world, a collaborative project which used a crowdsourced approach called OpenStreetMap was started. The data of OpenStreetMap is useful 
because firstly, the data is completely free with an open content license. Secondly, it is kept current because it is constantly being updated by the subscribed users who can also add points of interest important to them. OpenStreetMap has been used during earthquakes in Haiti as a reliable source of information (the map is named the Haiti Crisis Map). Finally, OpenStreetMap has the potential to benefit from volunteers from all over world including less developed regions, where obtaining data can be difficult for most commercial mapping companies. However, there can be map quality issues related to OpenStreetMap data, which may include:

- No moderation and a minimal imposed data model on data being uploaded

- Each individual has his own intents, purposes, motivations, knowledge, skill level and tools

- Digitisation errors

- Inaccurate mapping devices

- Uneven geographical spread of contributor efforts

- No proper interpretation of aerial photographs

Because of these potential issues, we tested OpenStreetMap data of India for quality. In addition, most of Indian map data (i.e. basic roads network and city names), are donated by Automotive Navigation Data (AND) (OpenStreetMap.org, 2013). It is possible that map data errors may also have come from the AND data source.

In this paper, we focus on assessing OpenStreetMap data for topological inconsistencies in the selected city of Punjab, India. These inconsistencies must be removed to ensure map data integrity. The structure of this paper is organised in different sections; the next section discusses about OpenStreetMap. Section 3 addresses topological inconsistencies and Section 4 elaborates on the methods used for the finding errors. Section 5 describes the results and the last section offers a conclusion.

\subsection{Crowdsourced OpenStreetMap Data}

The OpenStreetMap project was started in 2004 by Steve Coast with an idea to provide free and editable map data of the world. Two major driving forces behind the establishment and growth of OpenStreetMap have been restrictions on use or availability of map information across much of the world and the advent of inexpensive portable satellite navigation devices. OpenStreetMap is based on the concept of crowdsourcing, also called wikification of GIS, which encourages volunteers worldwide to contribute through the collection of geographic data.

OpenStreetMap has three main components, including:

- Nodes

- Ways

- Relations 
A Relation is a data structure that consists of other components such as nodes and ways to represent relationship between components. On 4th July, 2007, Automotive Navigation Data donated the entire road networks for India to OpenStreetMap to serve as a baseline to which volunteered data would be added.

\subsubsection{Why OpenStreetMap}

Proprietary map providers spend billions of dollars in creating and distributing maps. Crowdsourced maps such as OpenStreetMap produces a large volume of spatial data, with less effort and minimum cost (Goodchild, 2007). OpenStreetMap produces plentiful labelled data at no cost. This map data generation enables researchers to work on devising methods to use the data rather than collecting the data. Because many prior studies in algorithmic learning considered smaller training sizes due to traditional costs of labelling, there may be greater benefits to such learning techniques. Also Crowdsourcing offers coverage of many places around the world where there is little or no commercial coverage of map data provided by commercial vendors (e.g. even slum areas are often mapped by volunteers).

In addition, when other map providers try to monetise map data availability, OpenStreetMap respects communities, their work, and their privacy. The ownership of the content will always be with the user. OpenStreetMap uses a Creative Commons Attribution-ShareAlike 2.0 license, which allows the user to download data for any applications and/or analysis. Table 1 presents a few more benefits of using OpenStreetMap data as compared to proprietary map data providers.

\section{Table 1 Comparison of OpenStreetMap \& Proprietary Map Providers}

\begin{tabular}{ll}
\hline OpenStreetMap & Proprietary Map Provider \\
\hline Rendered maps or raw data & Rendered maps \\
No Developer Key & Developer Key \\
Multiple APIs & Single API \\
Several providers & Single provider \\
\hline
\end{tabular}

\subsubsection{OpenStreetMap Indian Users Statistics}

OpenStreetMap has a strong community of around 1.9 million contributors as shown in Figure 1. For India, the number of registered users is 2,877 . The total volume of data includes $7,561,749$ nodes, 433,747 ways and 6,094 relations in the map data. There are two types of edits, as shown in the Figure 2, v1 means objects are created, but are never modified (version 1) and 'last edit' represents objects modified at least one more time after creation. 


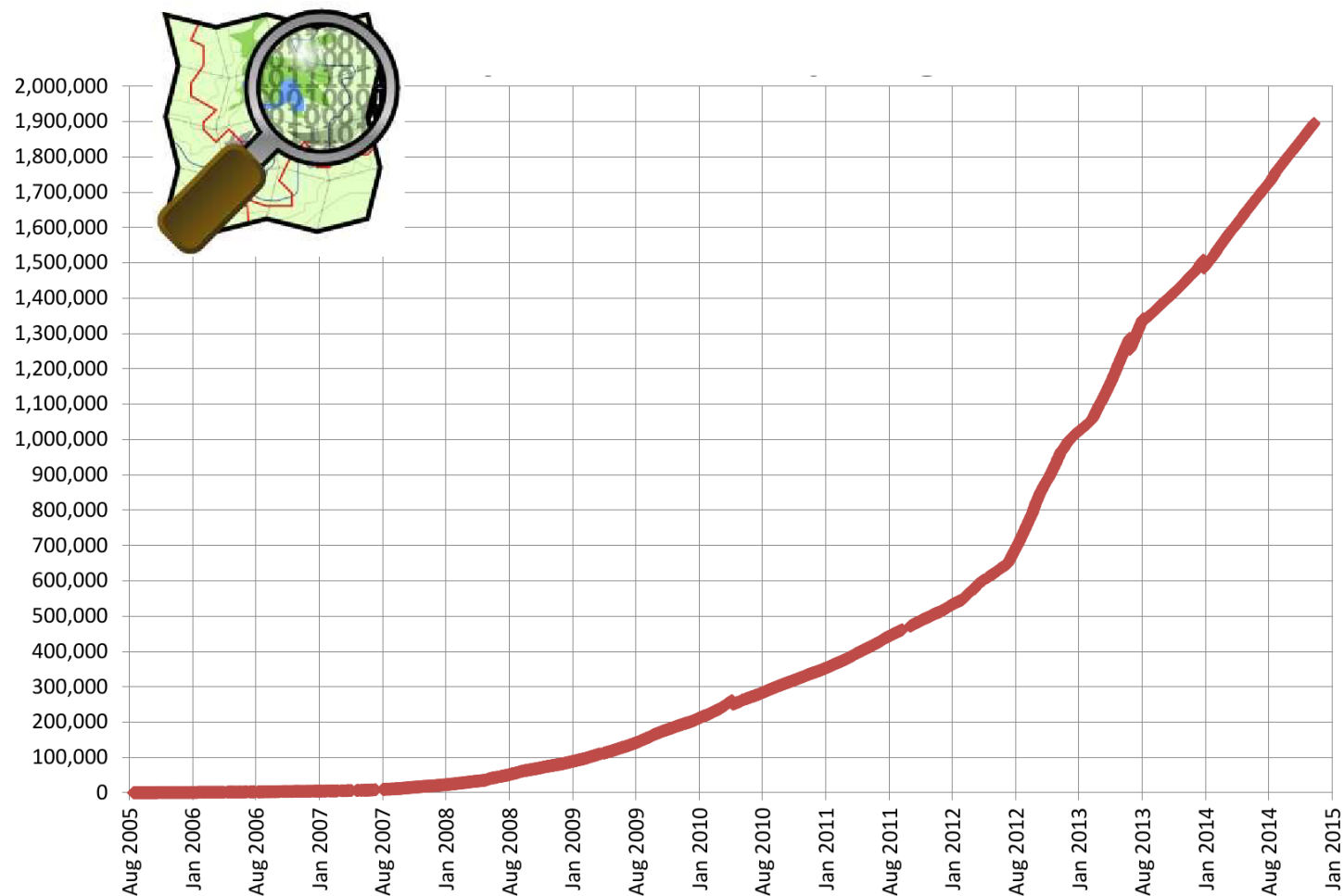

Figure 1. The statistics of registered users (OpenStreetMap.org, 2014b)

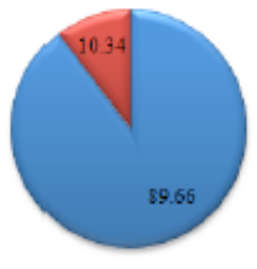

(a) Nodes

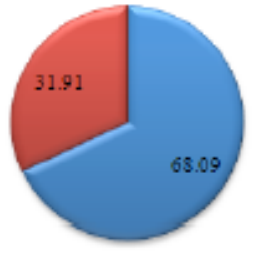

(b) Ways

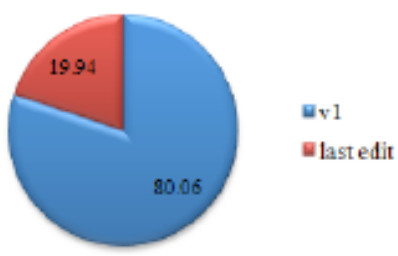

(c) Relations

Figure 2. OpenStreetMap Data of India (OpenStreetMap.org, 2014a)

\subsubsection{Map Data Contribution Methods}

The most common approach is recording map data using a Global Positioning System (GPS) receiver and editing the recorded information using one of the various freely available editors such as JOSM or Merkaator (Sehra et al., 2013). The users provide additional information about 
the collected data by adding attributes and storing the final results in the OpenStreetMap database. Users do not require any specialised GPS receiver for mapping; it has been tested that smartphones (Golicher, 2013) can be considered a reasonable mapping device. The accuracy of GPS devices on smartphones has been checked and found satisfactory. Microsoft Bing supports the OpenStreetMap project by providing various aerial images as background layers. The problem with such background layers is that these layers can be outdated and lack uniform coverage. Other web based tools include Potlatch2 and the iD editor as shown in Figure 3; only registered users can upload changes to OpenStreetMap.

\subsubsection{OpenStreetMap Data Repositories}

OpenStreetMap license terms allow users to download and distribute data. These data can be downloaded from the following repositories under the Creative Commons Attribution-ShareAlike 2.0 license terms:

1. Overpass API: Using this API, user can download a bounding box from a mirror of the OpenStreetMap database.

2. Planet OSM: This system provides regularly-updated copies of the complete OpenStreetMap database.

3. Geofabrik Downloads: This system provides regularly-updated extracts of continents, countries, and selected cities

4. Metro Extracts: This system provides extracts for major world cities and their surrounding areas can also be downloaded

The Indian map data for the proposed work was downloaded from the geofabrik repository in shapefile (.shp) format. Further test data for Ludhiana, Punjab was extracted after applying the clipping function available in Quantum Geographic Information System (QGIS) (Quantum, 2011). For clipping the test data, City, State administrative boundaries (polygons) were used as per Indian census 2011 data. 


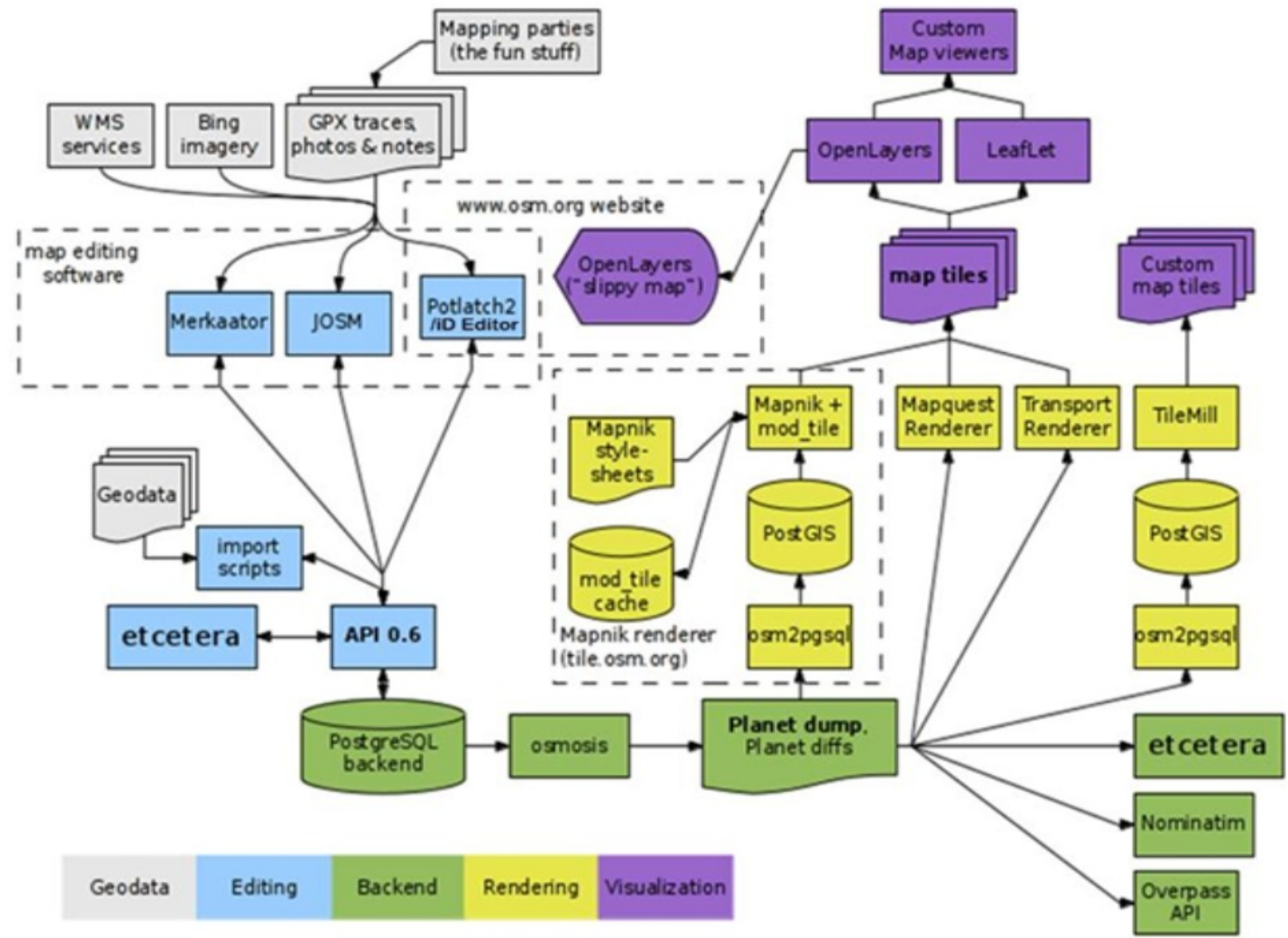

Figure 3 The OpenStreetMap architecture (OpenStreetMap, 2014).

\section{ELEMENTS OF OPENSTREETMAP DATA ASSESSMENT}

There are specific elements defined and used by the researchers for the assessment of OpenStreetMap data (Girres and Touya, 2010; Statistics Canada, 2013), which are given as follows:

1. Lineage - It describes the history of the spatial data, including descriptions of the source material from which the data were derived, and the methods of derivation. It also contains the dates of the source material, and all transformations involved in producing the final digital files or map products.

2. Positional accuracy - It is the spatial and geometric accuracy of the data. Positional accuracy of map data is represented as discrepancy between mapped point and reference point. Various methods are used for assessing the positional accuracy. Metrics used for spatial accuracy are root mean square error, standard deviation or confidence interval. 
3. Attribute accuracy - It refers to the accuracy of the quantitative and qualitative information attached to each feature (such as population for a population centre, street name, census subdivision name and code).

4. Topological consistency - It describes the trustworthiness of the topological and logical relationships between the dataset segments (Joksic and Bajat, 2004). These relations typically involve spatial data inconsistencies such as incorrect line intersections, polygons not properly closed, duplicate lines or boundaries, or gaps in lines. It deals with the structural integrity of a given data set based on formal framework for modelling of spatial data and relationships among objects. These types of errors must be corrected to avoid incomplete features and to ensure data integrity.

5. Completeness - It describes the completeness of objects and their attributes as compared to reference map data. It illustrates the commission of entities in spatial databases related to their number in the real world. It is an aspect of fitness-for-use. Fitness-for-use term is referred to decision making for accessing whether the database meets the requirements of a particular application.

All these elements depend upon the user mapping experience and tools used along with other support system also need reference datasets for assessment as done by most researchers. However, this paper focuses only on the topological inconsistencies which can be identified within the map dataset.

\section{TOPOLOGICAL CONSISTENCY}

Topological consistency describes the trustworthiness of the topological and logical relationships between the dataset segments (Joksic and Bajat, 2004). These relations typically involve spatial data inconsistencies such as incorrect line intersections, polygons not properly closed, duplicate lines or boundaries, or gaps in lines. It deals with the structural integrity of a given data set based on a formal framework for modelling of spatial data and relationships among objects. These types of errors must be corrected to avoid incomplete features and to ensure data integrity.

Topological errors, which occur during digitising and data exploration processes, are also known as semantic errors (Ubeda and Egenhofer, 1997). Topological errors exist due to violation of predefined topology rules. The most common topology errors in map data are shown in Figure 4 including:

- Duplicate Lines

- Overshoots

- Undershoots

- Micro Segments

- Pseudo Nodes

- Merge Adjacent Endpoints

- Self Intersection 

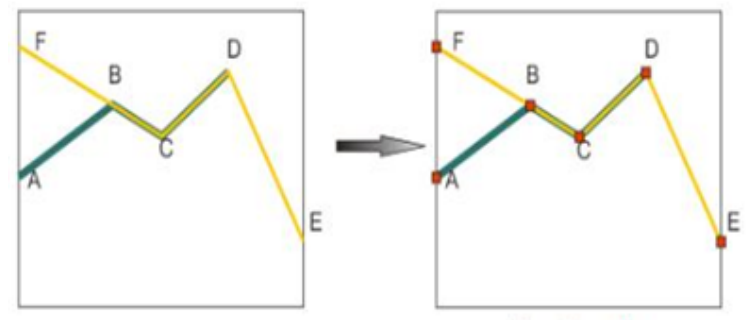

After intersecting

(a) Duplicate Nodes

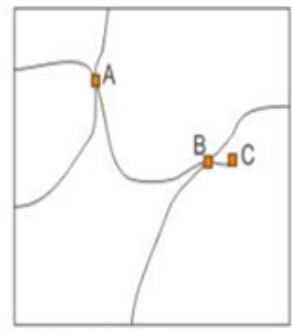

Dangle Tolerance

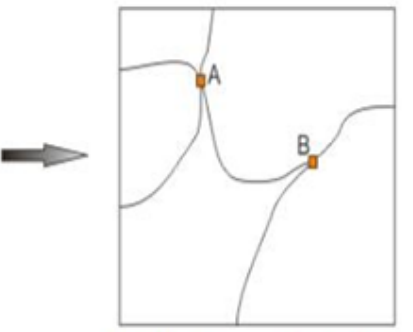

After cleanning the overshoot dangles

(b) Overshoots

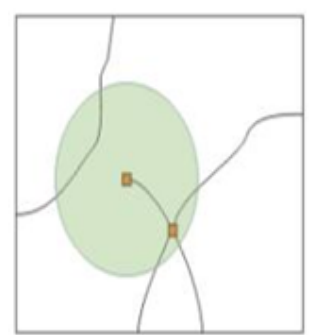

Dangle Tolerance

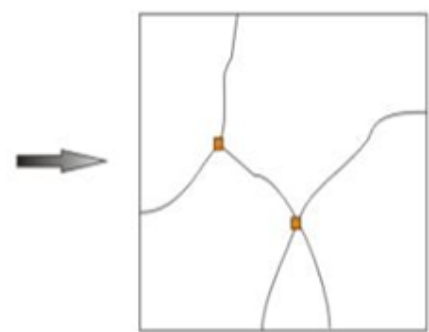

After extending the undershoots

(c) Undershoots

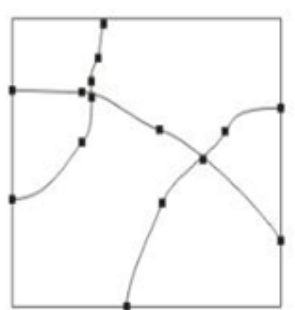

Fuzzy Tolerance -

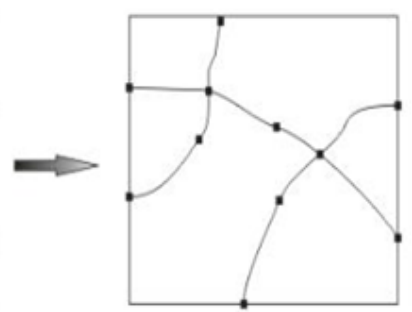

After cleanning the redundant vertices

(d) Redundant Vertices
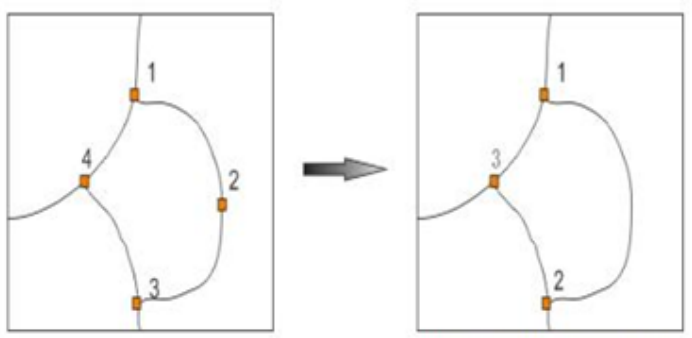

After merging the pseudo nodes

(e) Psuedo Nodes

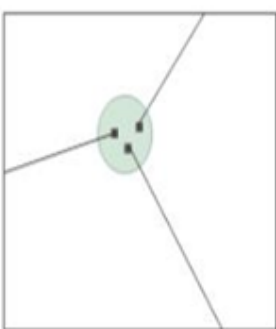

Fuzzy Tolerance

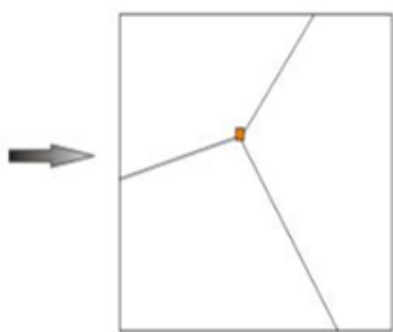

After merging the closed nodes (f) Merge Adjacent Endpoints

Figure 4. Various Topological Errors (Chang, 2008)

\section{METHODS OF TOPOLOGICAL ERROR DETECTION}

If a map is not topologically correct, then that map may not be useful in terms of navigation. OpenStreetMap data is not navigable in its original form (Neis et al., 2011). To test map data for topological errors, two map data file formats were used: one XML and the other Shapefile. Both 
files are used as required by the algorithm used for our analysis. The algorithm we used for the detection of topological errors is a plugin from OpenJump (Steiniger and Hunter, 2012). OpenJump is an open source Geographic Information System (GIS) written in the Java programming language. OpenJump is part of the Java Topology Suite (JTS), a Java API that implements robust geometric algorithms and provides a complete model for specifying 2-D linear Geometry. Another GIS software system we used included QGIS, which provides integration with many other packages (e.g. POSTGIS, GRASS, GeoServer and MapServer).

\subsection{Microsegment Vertices Detection}

Detecting microsegment vertices is the first step in the detection and cleaning process, because the algorithm uses a distance threshold to decide if two features should be snapped together or not. The algorithm used to detect microsegments chooses a point to be removed from the geometry if deformation is minimal (Michaël Michaud, 2014) e.g. micro segments have two vertices as shown in Figure 5. The problem with this algorithm is that it does not check if the vertex removed by the algorithm is also used by another geometry entity. This removal may break topology consistency. The Fig 6 shows the test data of Ludhiana city and detected microsegments found within it.

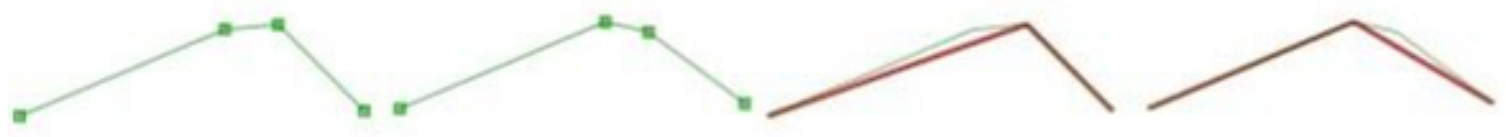

(a) Micro Segments

(b) After Cleaning of Micro Segments

Figure 5. Cleaning Microsegments

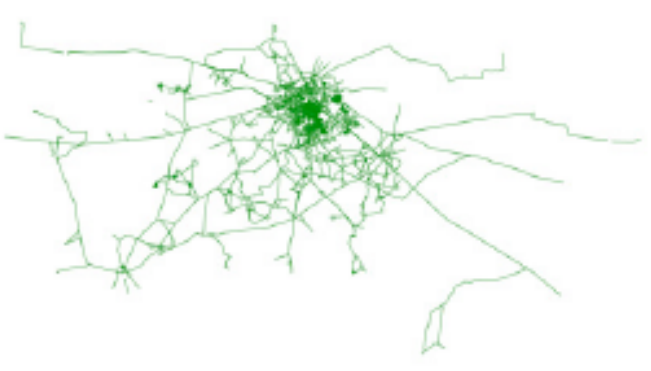

(a) Ludhiana City Data

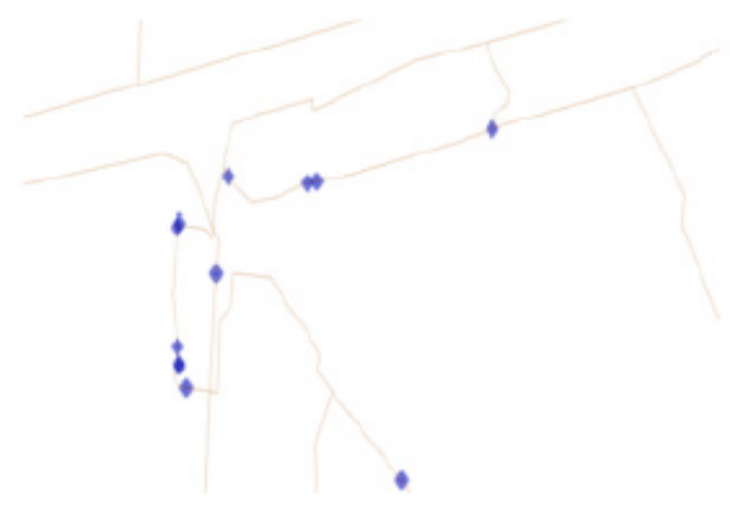

(b) Detected Mirco-segments 


\section{Figure 6. Micro-segment Detection}

\subsection{Network Topology Detection \& Correction}

This algorithm processes network datasets and detects topological errors such as node mismatches, undershoots, and overshoots as described previously and shown in Figure 4. It identifies nodes in networks based on a distance threshold between a node of the layer to be processes and a feature of the reference layer (Michaël Michaud, 2014).

\subsubsection{Detection of Nodes}

All nodes would be detected where the 1 st feature is less than 3 meters from a reference node; the 2nd and 3rd features are 1 meter from a segment, 2 meters from another one, and more than 3 meters from any vertex; the 4th feature is more than 3 meters from the feature, and less than 3 meters from a reference.

\subsubsection{Correction of Nodes}

The 1st and last features will be properly snapped to the reference, the 2nd and 3rd features will be also be snapped, but a vertex must be inserted into the reference layer for this to occur.

Another option available with this algorithm is to deal with degree $3+$ nodes of the processed layer. It would be useful to decrease the tolerance parameter for these nodes, because if they are not snapped on the reference layer, it is less likely that they should be snapped at all. The results obtained after processing the test data set are shown in Figure 7.

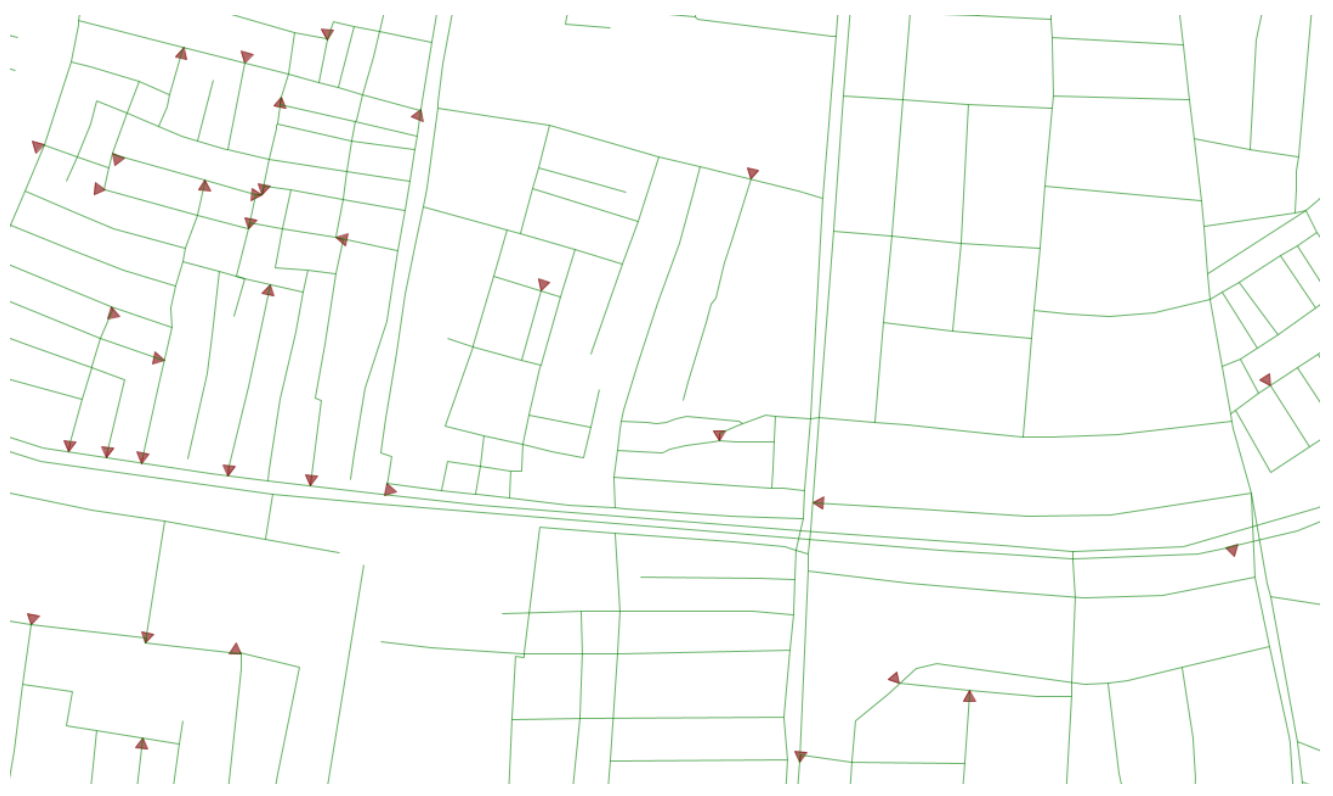




\section{Figure 7. Results from Network Cleaning Algorithm}

\subsection{Coverage Gaps Detection}

This algorithm is used for detection of defects like overlaps or gaps as shown in Figure 8. Nearly 95\% or more can be detected with this algorithm (Michaël Michaud, 2014).

- Segments are not topologically equivalent $\mathrm{AB}$ is not equal to $\mathrm{CD}$ and $\mathrm{AB}$ is not equal to $\mathrm{DC}$

- Minimum distance between segments is less than the user-defined tolerance

- The angle between both segments is less than the user-defined tolerance

- The orthogonal projection of each segment on the matched one is not null

\subsection{Bad Features in OpenStreetMap}

We detect bad features in OpenStreetMap data using a Geometry Metric validation algorithm in Openjump and QGIS. It is tested based on the following rules enforced on the data:

- $\quad$ Basic topology

- Disallow repeated consecutive points

- Polygon orientation

- Minimum segment length (0.001 is taken here)

- Minimum angle in degrees (1.0 is taken)

- Minimum polygon area (0.01)

- Geometries are simple (i.e., do not self-intersect)

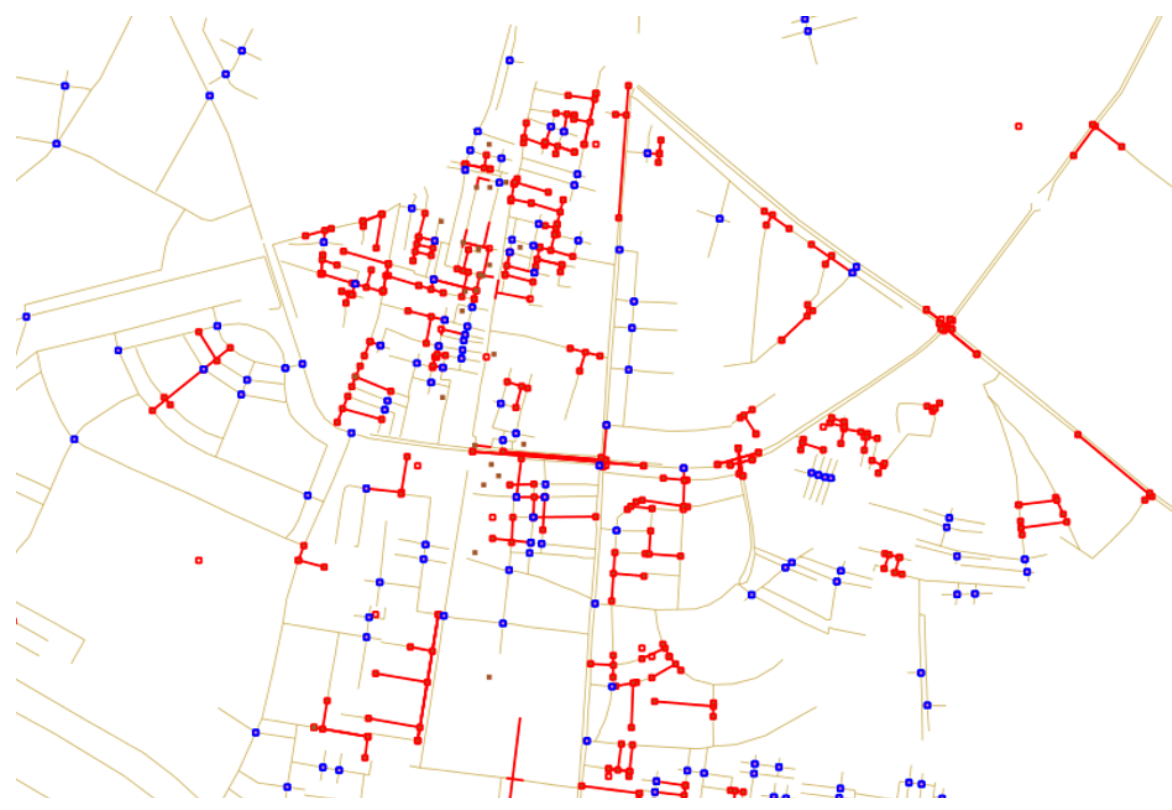




\section{Figure 8. Outcome of Coverage Gaps Algorithm}

\section{RESULTS}

The test data of Ludhiana city, located in Punjab was extracted using the QGIS clipping function on the OpenStreetMap data of India. The extracted data included a total of 3,210 map segments and 20,550 nodes. These data were then processed using various error-detection algorithms as described above, which produced the following results.

- Micro-segment detection algorithm: 37 micro-segments detected

- Network cleaning algorithm: 309 mismatched features, 618 points

- Coverage gap algorithm (Parameters: distance tolerance $=1.0$, angle tolerance $=22.5$, without Fence): gap segments of 12 features and 24 points, overlaps of 959 features and 8607 points

- $\quad$ Feature mismatch analysis: 32 bad features and 5 points

- QGIS topology checker: 5034 dangling errors, one duplicate node, 90 geometry errors, 24 mulitpart-geometry errors, and 348 pseudo nodes

\section{CONCLUSION}

OpenStreetMap is the world leader in collecting map data contributed by volunteer crowdsourced users. However, these diverse users are of different backgrounds and have varying level of mapping experience. Additionally, this human computation system uses loose coordination and no top-down quality assurance processes. This makes map data more vulnerable to errors and incomplete coverage. Nonetheless, OpenStreetMap demonstrates the increasing relevance of open and crowd-sourced data for commercial use. Indeed, there are over 60 companies using OpenStreetMap data commercially, which would benefit from improvements in data quality assessment and assurance.

As described earlier (and illustrated in Figure 2), most of the data in the study set, originated from AND, signifying that the majority of the map data was never modified by OpenStreetMap users. This indicates that relatively less volunteered mapping activity occurred in this area. Due to the constantly changing roadway infrastructure of this area, an accurate map is highly dependent on user-generated edits. Therefore, this particular dataset may be more prone to errors relative to OpenStreetMap data from areas with either a more stable roadway infrastructure or a more active user base of OpenStreetMap contributors.

During this work, we used data from the city of Ludhiana in Punjab, India and processed these data using various algorithms. We found that map data within this test dataset contain many topological errors. We conclude that such map data for the city is not mature enough to be used for navigational purposes. Future work must focus on removing topological errors from the OpenStreetMap data of Punjab in addition to the detection of such errors. Taken together, our 
exploration of error detection algorithms in concert with data that has been volunteered by human contributors illustrates a human computation system that yields significant benefits by enabling broader mapping scope but that may also derived improved map accuracy from greater machinebased involvement in the form of error-detection and correction using methods such as those employed and described herein. 


\section{REFERENCES}

Ather, A. (2009). A Quality Analysis of OpenStreetMap. (2009). ftp://ftp.cits.rncan.gc.ca/pub/cartonat/Reference/VGI/ Dissertation-OpenStreepMap-Quality-Aather-2009.pdf

Chang, K. T. (2008). Introduction to GIS. McGraw-Hill. Fischer, F. (2008). Collaborative mapping-How wikinomics is manifest in the geo-information economy. Geoinformtics 11, 2 (2008), 28-31. http://www.gisaci.upol.cz/filesftp/Geoinformatics-02-2008.pdf

Girres, J and Touya, G. (2010). Quality assessment of the French OpenStreetMap dataset. Transaction in GIS 14 (2010), 435-459. http://onlinelibrary.wiley.com/doi/10.1111/j.1467-9671.2010.01203.x/abstract

Golicher, D. (2013). Accuracy of an Android cell phone GPS in the UK. (2013). http://duncanjg.wordpress.com/2011/05/08/accuracy-of-an-android-cell-phone-gps-in-the-uk/

Goodchild, M. (2009). NeoGeography and the nature of geographic expertise. Journal of Location Based Services 3,2 (2009), 82-96. http://www.tandfonline.com/doi/abs/10.1080/17489720902950374

Goodchild, M. F. (2007). in the World of Web 2.0. International Journal 2 (2007), 24 ?32. http://www.geoinformatics.cn/wp-content/uploads/citizensasvoluntarysensors.pdf

Haklay, M, Singleton, A, and Parker, C. (2008). Web mapping 2.0: The neogeography of the GeoWeb. Geography Compass 2, 6 (2008), 2011-2039. http://onlinelibrary.wiley.com/doi/10.1111/j.1749-8198.2008.00167.x/full

Howe, J. (2006). The rise of crowdsourcing. Wired magazine 14, 6 (2006), 1-4. http://sistemas-humanocomputacionais.wikidot.com/local--files/capitulo:redes-sociais/Howe_The_Rise_of_Crowdsourcing.pdf

Hudson-Smith, A, Crooks, A, Gibin, M, Milton, R, and Batty, M. (2009). NeoGeography andWeb 2.0: concepts, tools and applications. Journal of Location Based Services 3, 2 (2009), 118-145.

http://www.tandfonline.com/doi/abs/10.1080/17489720902950366

Joksic, D and Bajat, B. (2004). Elements of spatial data quality as information technology support for sustainable development planning. 11 (2004), 77-83. http://www.doiserbia.nb.rs/Article.aspx?ID=1450-569X0411077J

Kounadi, O. (2009). Assessing the quality of OpenStreetMap data. MSc Dissertation. University College of London, Department of Civil, Environmental And Geomatic Engineering. ftp://ftp.cits.nrcan.gc.ca/pub/cartonat/Reference/VGI/Rania_OSM_dissertation.pdf

Michaël Michaud, . (2014). Personal Website : Topology Library. (2014). http://geo.michaelm.free.fr

Neis, P, Zielstra, D, and Zipf, A. (2011). The street network evolution of crowdsourced maps: OpenStreetMap in Germany 2007-2011. Future Internet 4, 1 (2011), 1-21. http://www.mdpi.com/1999-5903/4/1/1

Newcomb, D. (2014). Telenav's switch to Open Source Mapping allows for more frequent software updates and traffic reports. (2014). http://www.pcmag.com/article2/0,2817,2458392,00.asp

OpenStreetMap, . (2014). Component Overview of OpenStreetMap. (2014). http://wiki.openstreetmap.org/wiki/Component_overview

OpenStreetMap.org. (2013). Automotive Navigation Data. (2013). http://wiki.openstreetmap.org/wiki/AND_Data

OpenStreetMap.org. (2014)a. OpenStreetMap ODbL acceptance and user ranks for the region of india. (2014). http://odbl.de/india.html 
OpenStreetMap.org, . (2014)b. OpenStreetMap Statistics. (2014).

http://www.openstreetmap.org/stats/data_stats.html

O'Reilly, T. (2005). What IsWeb 2.0: Design Patterns and Business Models for the Next Generation of Software. In O' Reilly Media:. Cambridge, USA. http://oreilly.com/web2/archive/what-is-web-20.html

Quantum, G. (2011). Development Team (QGIS). 2011. Quantum GIS Geographic Information System, Version 1.7.0. Open Source Geospatial Foundation Project. Vancouver. British Columbia, Canada. qgis. osgeo. org (2011).

Schmitz, S, Zipf, A, and Neis, P. (2008). New Applications based on collaborative geodata?the case of Routing. In XXVIII INCA international congress on collaborative mapping and space technology, Gandhinagar, India (2008). http://koenigstuhl.geog.uni-heidelberg.de/publications/bonn/conference/cmap2008.cartography-bonn.subm.pdf

Sehra, S. S, Singh, J, and Rai, H. S. (2013). Assessment of OpenStreetMap Data - A Review. International Journal of Computer Applications 76, 16 (2013), 17-20. DOI:http://dx.doi.org/10.5120/13331-0888

Statistics Canada, . (2013). Spatial data quality elements. (2013). http://www.statcan.gc.ca/pub/92-195x/2011001/other-autre/qua-eng.htm

Steiniger, S and Hunter, A. J. S. (2012). OpenJUMP HoRAE-A free GIS and toolbox for home-range analysis. Wildlife Society Bulletin 36, 3 (2012), 600-608. DOI:http://dx.doi.org/10.1002/wsb.168

Turner, A. (2006). Introduction to Neogeography. O’Reilly Media, MA, USA (2006). http://shop.oreilly.com/product/9780596529956.do

Ubeda, T and Egenhofer, M. (1997). Topological error correcting in GIS. In Advances in Spatial Databases, Michel Scholl and Agnès Voisard (Eds.). Lecture Notes in Computer Science, Vol. 1262. Springer Berlin Heidelberg, 281-297. http://dx.doi.org/10.1007/3-540-63238-7_35. 